\title{
Tailoring the energy landscape in quasi-2D halide perovskites enables efficient green light emission
}

\author{
Li Na Quan, ${ }^{1,2 \dagger}$ Yongbiao Zhao, ${ }^{3 \dagger}$ F. Pelayo García de Arquer, ${ }^{1 \dagger}$ Randy Sabatini, ${ }^{1}$ Grant \\ Walters, ${ }^{1}$ Oleksandr Voznyy, ${ }^{1}$ Riccardo Comin, ${ }^{1}$ Yiying Li, ${ }^{3}$ James Z. Fan, ${ }^{1}$ Jun Pan, ${ }^{4}$ Mingjian \\ Yuan, ${ }^{1}$ Osman M. Bakr, ${ }^{4}$ Zhenghong Lu, ${ }^{3 *}$ Dong Ha Kim, ${ }^{2}$ Edward H. Sargent ${ }^{1}$ * \\ ${ }^{1}$ Department of Electrical and Computer Engineering, University of Toronto, 10 King's College \\ Road, Toronto, Ontario, M5S 3G4, Canada. \\ ${ }^{2}$ Department of Chemistry and Nano Science, Ewha Woman's University, 52, Ewhayeodae-gil, \\ Seodaemun-gu, Seoul 03760, Korea. \\ ${ }^{3}$ Department of Materials Science and Engineering, University of Toronto, 184 College Street, \\ Toronto, Ontario M5S 3E4, Canada. \\ ${ }^{4}$ Division of Materials Science and Engineering, King Abdullah University of Science and \\ Technology, Thuwal 23955-6900, Kingdom of Saudi Arabia \\ $\uparrow$ These authors contributed equally to this work. \\ E-mail: ted.sargent@,utoronto.ca; dhkim@ewha.ac.kr; zhenghong.lu@utoronto.ca
}

\begin{abstract}
Organo-metal halide perovskites are a promising platform for optoelectronic applications in view of their excellent charge transport and bandgap tunability. However, their low photoluminescence quantum efficiencies, especially in low excitation regimes, limit their efficiency for light emission. Consequently, perovskite light emitting devices are operated under high injection, a regime under which the materials have so far been unstable. Here we show that, by concentrating photoexcited states into a small subpopulation of radiative domains, one can achieve a high quantum yield even at low excitation intensities. We tailor the composition of quasi-2D perovskites to direct energy transfer into the lowest-bandgap
\end{abstract}


minority phase, faster than it can be lost to non-radiative centres. The new material exhibits $60 \%$ photoluminescence quantum yield at excitation intensities as low as 1.8 $\mathrm{mW} / \mathrm{cm}^{2}$, yielding a ratio of quantum yield to excitation intensity of $0.3 \mathrm{~cm}^{2} / \mathrm{mW}$; this represents a two-orders of magnitude decrease in the excitation power required to reach high efficiency compared to the best prior reports. Using this strategy, we report LEDs with EQEs of $7.4 \%$ and a high luminescence of $8400 \mathrm{~cd} / \mathrm{m}^{2}$.

Organo-metal halide perovskites (OHP) have emerged as a new class of optoelectronic materials for solar cells ${ }^{123}$, optically-pumped lasers ${ }^{456}$, and light emitting diodes (LEDs) 78910 11. However, their low exciton binding energy ${ }^{12} 1314$, as well as their high electron and hole mobilities, lead to poor photoluminescence quantum yields (PLQYs) at low excitation regimes 15. In order to attain useful PLQYs, high excitation photon fluences must be supplied, and this ultimately results in compromised photostability that limits light-emission applications ${ }^{16}$.

Organic-inorganic hybrid perovskites have an $\mathrm{ABX}_{3}$ three-dimensional (3D) lattice framework that, through ion modification, can be spectrally tuned ${ }^{17}$. The low exciton binding energy in these systems results in a free-charge character in crystalline domains that slows radiative recombination ${ }^{14}$. Exciting the materials into a high injection regime is required to attain moderate light-emission efficiency. This, on top of the limited stability of these materials ${ }^{18}$, can produce unstable operation and fast degradation in light-emitting applications ${ }^{19}$.

In contrast, lower dimensionality organic-inorganic hybrid perovskites are characterized by inorganic layers spaced using organic ligands ${ }^{20}$. This provides an excellent combination of structural and photophysical properties, such as the ability to tune the material bandgap depending on the dimensionality ${ }^{21,22}$, increased exciton binding energy and higher stability compared to $3 \mathrm{D}$ counterparts ${ }^{18}$. Depending on the number of perovskite layers sandwiched 
between the organic linkers, these type of OHPs are termed as $2 \mathrm{D}$ (e.g, a single $\mathrm{PbBr}_{2}$ layer) or quasi-2D (a distribution of layers with different thicknesses) perovskites.

Low-dimensional perovskites are particularly attractive for visible light emission applications, and LEDs based on quasi-2D perovskites have been actively explored. However, these devices show low efficiency (below 1\%) and limited photo-stability (Table 1) ${ }^{23,24}$.

The radiative properties of quasi-2D perovskite systems based on $\mathrm{PEA}_{2}(\mathrm{MA})_{\mathrm{n}-1} \mathrm{~Pb}_{\mathrm{n}} \mathrm{I}_{3 \mathrm{n}+1}$ ( $\mathrm{PEA}=$ phenylethylammonium; $\mathrm{MA}=\mathrm{CH}_{3} \mathrm{NH}_{3}=$ methylammonium) have recently been reported for near-infrared LED applications ${ }^{25}$. The dimensionality of this material was tuned by varying the ratio of methylammonium iodide (MAI) to PEAI. This created a distribution of domains with varying numbers of perovskite layers centered on an average value $<n>$. An impressive PLQY enhancement was achieved in the low $<n>$ regime. This was attributed to an inhomogeneous energy landscape that favoured funnelling of energy into domains with lower bandgap (higher dimensionality). A quantitative model of this phenomenon, together with a design strategy to unlock further advances in performance, remains to be reported.

Herein we demonstrate the prerequisites for an efficient energy funneling mechanism that gives rise to high photoluminescence yields in quasi-2D perovskite systems. We find that only when energy is funneled from high bandgap domains into a small sub-population of lower bandgap domains does radiative recombination outcompete non-radiative recombination. Only then does the high charge concentration in these domains modulate charge-recombination kinetics. In particular, we found that the distribution of these domains is crucial for achieving high PLQY and LED performance.

Based on these findings, we then developed a fabrication strategy to control the domain distribution in quasi-2D perovskites. Specifically, we modulate the domain distribution in 
$\mathrm{PEA}_{2}(\mathrm{MA})_{\mathrm{n}-1} \mathrm{~Pb}_{\mathrm{n}} \mathrm{Br}_{3 \mathrm{n}+1}$ perovskites through their composition, and solvent engineering during crystallization. In agreement with the model, only when the quasi-2D films are comprised of a distribution of different bandgap domains does highly efficient light-emission occur.

The energy-landscape-engineered quasi-2D perovskite system exhibits high photoluminescence yields at remarkably low excitation intensities $\left(60 \%\right.$ at $1.8 \mathrm{~mW} / \mathrm{cm}^{2}$, corresponding to a ratio of quantum yield to excitation intensity of $0.3 \mathrm{~cm}^{2} / \mathrm{mW}$ ). This represents an over two-orders-of-magnitude decrease in the excitation density at which the quantum yield rises above the 50\% mark when compared to previously-reported solutionprocessed perovskite PLQYs. The energy-landscape-engineered quasi-2D platform is stable over the course of a week when stored under ambient conditions (Figure S12).

Using these materials, we fabricated LED devices with external quantum efficiencies (EQEs) of $7.4 \%$ accompanied by high luminescence brightness $\left(8400 \mathrm{~cd} / \mathrm{m}^{2}\right)$. These also feature notable improvements in stability compared to conventional $\mathrm{MAPbBr}_{3}$ perovskites.

\section{Results and discussion}

In conventional bulk 3D perovskites (Fig. 1a), excited charges diffuse faster than they recombine radiatively, ultimately becoming trapped at grain boundary defects. In lower dimensional systems, such as quasi-2D OHPs, a combination of free charges and excitons coexist in smaller grains, which increases the radiative recombination probability compared to the bulk-3D scenario (Fig. 1b). However, surface and interface defects can still dominate because of the smaller grain sizes and the extended interfaces ${ }^{26} 27$. We hypothesized that, if the energy landscape in this material system was modulated in such a way that energy transfer could be fast enough to outpace trapping and subsequent non-radiative recombination, photoexcited states would be concentrated in small radiative domains where bimolecular radiative recombination 
would be enhanced because of the higher concentration (Fig. 1c). This would in turn result in high luminescence yields, even in low injection regimes.

To gain insight into the conditions under which energy transfer is sufficient to enhance radiation efficiency, we developed a stochastic Monte Carlo model that accounted for charge recombination and transfer processes (Fig. 1d-e, Suppl. section S1). The dynamics of the excited population $(N)$, following impulsive photoexcitation, can be described by the rate equation:

$$
\frac{d N}{d t}=-N\left(A+B N+C N^{2}\right)+\phi(t)
$$

where $A, B, C$ are material-specific coefficients related respectively to Shockley-ReadHall (trap) recombination $\left(1^{\text {st }}\right.$ order$)$, bimolecular photoluminescence $\left(2^{\text {nd }}\right.$ order$)$, and Auger recombination $\left(3^{\text {rd }}\right.$ order $) . \phi(t)$ represents the rate of exciton generation per volume ${ }^{28}$.

We first modelled the energy transfer and recombination yield of a flat energy landscape material (Fig. 1f, supplementary section S1). Under these conditions we observe no preferential energy accumulation (Fig. 1f inset) and, as a consequence, trapping becomes the dominant loss mechanism (Fig. S2).

Energy-landscape-engineered multidomain materials, on the other hand, display a significantly different behavior (Fig. 1g). For example, in a ternary system consisting of $<n>=$ $\left\{n_{1}, n_{2}, n_{3}\right\}$, (see suppl. section $\mathrm{S} 1$ for details), excitons sequentially funnel from higher to lower bandgap domains, ultimately concentrating in final acceptor domains $\left(n_{3}\right)$ (Fig. $1 \mathrm{~g}$ inset). In this scenario, photon emission outcompetes trapping within the initial 10 ps (Fig. S11) as the strong charge concentration favours bimolecular radiative recombination (eq. 1).

This downward energy cascade is expected to reduce the typically high optical pump powers and driving voltages required for efficient light emission in OHP systems due to the 
improved radiative efficiency. This is revealed by the computed photoluminescence quantum yield (PLQY) as a function of pump power intensity in different materials systems (Fig. 1h). Depending on the concentration coefficient $f$-where $f$ denotes the effective increase of charge density in the final radiative domains compared to a flat-energy-landscape scenario - we observe a two orders-of-magnitude reduction in the pump power required to achieve high PLQY as $f$ increases from 1 to 10 . We conclude that energy funneling in engineered landscapes can be exploited to improve the radiative properties of otherwise non-ideal materials, leading to efficient light emission achievable at lower driving powers.

With these concepts in mind, we sought to define experimentally the energy landscape and energy flow dynamics in different OHP material systems. We synthesized quasi-2D bromide perovskites $\mathrm{PEA}_{2}(\mathrm{MA})_{\mathrm{n}-1} \mathrm{~Pb}_{\mathrm{n}} \mathrm{Br}_{3 \mathrm{n}+1}$ at a judiciously selected stoichiometry (Supplementary Fig. $\mathrm{S} 2)^{18,25}$. We employed different solvents to control perovskite formation in a fast crystallization single-step spin-coating method ${ }^{29}$. We took the view that the different boiling point of toluene $\left(110^{\circ} \mathrm{C}\right)$ and chloroform $\left(61^{\circ} \mathrm{C}\right)$ would result in different crystallization kinetics and resulting domain distributions. The high boiling point of toluene would favour the formation of more uniform domains (flat energy landscape) whereas chloroform, with faster evaporation, would lead to the formation of smaller clusters with different bandgaps (Supplementary Fig. S4 and S5).

We used ultrafast transient absorption spectroscopy (TA) measurements, to monitor the dynamics of energy transfer and thereby reveal the differences between these material configurations (Fig. 2). In the case of the flat energy landscape of standard quasi-2D perovskites, no energy migration is observed. A single bleach decay is recorded that coincides with the ground state bleach (GSB) and the excitonic peak obtained by linear absorption measurements 
(Fig. 2a-b). The extracted domain distribution is in this case dominated by a single domain component (Fig. 2c).

Energy-landscape-engineered perovskites behave quite differently (Fig. 2d-f). Several GSBs are observed, and the decay kinetics of the different GSB peaks correlate well with the notion of energy transfer. ${ }^{30}$ The GSB maxima are sequentially delayed for lower energy features, indicative of domains that receive charges from (larger bandgap) domains. The energy transfer is

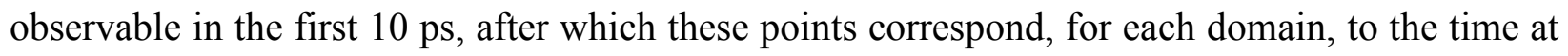
which the population in each lower domain decays faster than it grows from energy funneling. The decay kinetics are slower for lower energy features (acceptor domains). These facts, together, indicate that energy is being funneled from wider to narrower bandgap grains. The extracted domain distribution is in this case comprised by a set of components with different bandgaps that favour energy concentration into the emitting domain, (Fig. 2e). Signatures of energy transfer in landscape-engineered perovskites are also evident in time-resolved PL emission studies, which show ultrafast (ps) and slow (ns) transfer components (Supplementary Fig. 10).

We then sought to apply our Monte-Carlo model to fit the energy transfer kinetics in different landscape-engineered configurations to provide insights into the rates of the different processes involved (Fig. 3a). The different rise, maxima, and fall times for the various GSBs are evidence of energy transfer between the different domains which are in in good agreement with the theoretical predictions. Our model elucidates transfer rates on the order of $\mathrm{ps}^{-1}$ between the different perovskite domains, and estimates the instantaneous contributions of radiative, trap and Auger mechanisms (Supplementary Fig. S11). Photoexcited states' lifetimes are shorter in wider- 
bandgap grains, as these are the most likely to be transferred to narrower-bandgap grains over $\sim 10$ ps (Table 2).

With these findings in mind, we then sought to further improve the final radiative efficiency, and therefore pursued control of the distribution of the different domains. We took the view that optimizing the concentration of the $n=5$ emitting domains would allow a more efficient, graded funneling, where the majority of the charges are concentrated in $n=5$ domains rather than trapped or emitted in higher bandgap clusters before concentration.

To do so we reduced the quantity of organic PEABr and $\mathrm{MABr}$ ligands involved in the perovskite synthesis, seeking to promote the formation of $n=5$ domains. As revealed by transient absorption, the resulting films exhibited a different, broader distribution of $n$ domains with a higher $n=5$ contribution (Supplementary Fig. S9). We input the extracted domain distribution into our model, which yields good agreement with the experimental TA decay traces (Fig. 3b). The decay kinetics reveal a faster charge injection from the donor to the acceptor domains, which we attribute to the more graded domain distribution that increases the probability of sequential energy transfer.

We then sought to assess experimentally how efficient energy transfer could lower the power at which high PLQY could be attained, and studied PLQY as a function of pump power for the different types of perovskite systems (Fig. 3c). As predicted, energy-landscapeengineered quasi-2D perovskites show a substantially higher PLQY compared to flat-landscape, bulk (MAPbBr 3 ) and $\mathrm{PEA}_{2} \mathrm{PbBr}_{4}(2 \mathrm{D})$ perovskites (Supplementary Fig. S8).

Graded landscape $<n>=5$ perovskites show a prominent PLQY of $\sim 60 \%$ at an excitation power density as low as $1.8 \mathrm{~mW} / \mathrm{cm}^{2}$ (Fig. 3d). This yields a PLQY to pump power density ratio of $0.3 \mathrm{~cm}^{2} / \mathrm{mW}$, a two-orders-of-magnitude improvement over prior perovskite systems (Table 
3). Notably, landscape-engineered quasi-2D perovskite films exhibit significantly superior photostability compared to their bulk 3D counterparts, retaining a high PLQY in excess of $80 \%$ of the initial value over the course of a one week initial study (Supplementary Fig. 12).

We then translated the low operating powers of refined landscape-engineered quasi-2D perovskite materials into LED applications, and built LED devices employing $<n>=3$ (engineered landscape), $<n>=5$ (graded optimized landscape), and bulk $\mathrm{MAPbBr}_{3}$ (flat landscape) photoactive layers (Fig. 4a). Cross-sectional SEM indicates the presence of two uniform layers: a hole injection layer of PEDOT:PSS (A14083) $(\sim 10 \mathrm{~nm})$ coated with a dense perovskite emitter layer $(\sim 130 \mathrm{~nm}) .100 \mathrm{~nm}$ of TPBi was coated to provide an electron injection layer, followed by a $\mathrm{LiF} / \mathrm{Al}(1 / 100 \mathrm{~nm})$ electrode on top (Fig. 4b). Under forward bias, electrons and holes are injected from the cathode/electron injection layer (TPBi) and anode/hole injection layer (PEDOT:PSS) respectively, and are efficiently transferred to the perovskite, where they concentrate into the emitting domains to recombine radiatively.

Flat landscape bulk perovskite devices exhibit low performance with a $0.8 \%$ EQE and $33.7 \mathrm{~cd} / \mathrm{m}^{2}$ luminance (Fig. $4 \mathrm{c}$ and d). This is expected in view of their lower PLQY (Fig. 3f).

Devices based on landscape-engineered quasi-2D perovskites, on the other hand, show improved optoelectronic characteristics. $<n>=3$ devices exhibit reduced turn-on voltages and an order-of-magnitude increase in EQE and luminance (up to $3.8 \%$ and $1000 \mathrm{~cd} / \mathrm{m}^{2}$ ) (Fig. $4 \mathrm{c}$ and d). Graded $<n>=5$ devices display a significant enhancement in EQE (7.4\%) and luminance (8400 $\mathrm{cd} / \mathrm{m}^{2}$ ), in agreement with the trends observed in PLQY (Supplementary Fig. S13 and S15). The EL spectra of these devices closely match the PL spectra, preserving a narrowband emission (Supplementary Fig. S14). Large area devices $\left(6.54 \mathrm{~cm}^{2}\right)$ based on graded $<n>=5$ perovskites were also fabricated (Fig. 4d inset) and show high brightness with strong optical uniformity. 


\section{Conclusion}

We demonstrated the conditions required for efficient radiative recombination in low dimensional perovskite systems. We found that when the energy landscape in the perovskite is modulated in such a way that energy transfer outpaces non-radiative recombination, concentrating charges into a subpopulation of radiative domains, efficient light emission take place. We devised a perovskite synthesis and crystallization method whereby domain distribution could be controlled. By optimizing the domain distribution, we achieved a record PLQY of $60 \%$ at low excitation fluences $\left(1.8 \mathrm{~mW} / \mathrm{cm}^{2}\right)$ for solid films at green wavelengths. This represents the highest maximum yield-to-driving-power reported for organo-metal halide perovskite materials $\left(0.3 \mathrm{~cm}^{2} / \mathrm{mW}\right)$. We translated these findings into LED devices with high EQEs $(7.4 \%)$ and high luminescence $\left(8400 \mathrm{~cd} / \mathrm{m}^{2}\right)$ at low threshold voltages.

\section{Materials and Methods}

\section{Perovskite film fabrication.}

The quasi-2D and $\mathrm{MAPbBr}_{3}$ perovskites precursors were prepared by dissolving specific stoichiometric quantities of lead bromide $\left(\mathrm{PbBr}_{2}, 99.98 \%\right.$ Alfa-Aesar), methylammonium bromide (MABr, Dyesol) and phenylethylammonium bromide (PEABr, Dyesol) in DMSO solvent. The resulting solution was filtered using a PTFE syringe filter $(0.2 \mu \mathrm{m})$ before deposition. The precursor solution was coated onto the substrate via a consecutive two-step spin coating process at 1000 r.p.m. and 5000 r.p.m. for 10 and 60 seconds, respectively. During the second spin step, $100 \mu \mathrm{L}$ of chloroform (or toluene) were deposited onto the substrate. The resulting films were then annealed at $90^{\circ} \mathrm{C}$ for 5 minutes for better crystallization.

\section{Light-Emitting Diodes fabrication.}


A PEDOT:PSS (Clevios ${ }^{\mathrm{TM}}$ PVP A14083) layer was spin-coated on oxygen-plasma-treated patterned ITO-coated glass substrates, then annealed on a hot plate at $150^{\circ} \mathrm{C}$ for 20 minutes in air. Perovskite precursor solutions were spin-coated onto the PEDOT:PSS via the two-step fast crystallization spin-coating method that was described above. TPBi $(100 \mathrm{~nm})$ and LiF/Al electrodes $(1 \mathrm{~nm} / 100 \mathrm{~nm})$ were deposited using a thermal evaporation system through a shadow mask under a high vacuum of less than $10^{-4} \mathrm{~Pa}$. The device active area was $12.2 \mathrm{~mm}^{2}$ as defined by the overlapping area of the ITO and Al electrodes. Unpatterned ITO substrates (1 by 1 inch) were used for the large-area devices. All devices were tested under ambient condition.

\section{AFM measurements.}

Atomic force microscopy (AFM) was used to characterize sample morphology. An Asylum Research Cypher AFM was operated in AC mode in air to obtain topographical and phase images. ASYELEC-02 silicon probes with titanium-iridium coatings from Asylum Research were used for all imaging. The typical spring constant of the probes is $42 \mathrm{~N} / \mathrm{m}$.

\section{XRD measurements.}

XRD measurement on oriented films were conducted on a Panalytical X'Pert Pro diffractometer with a Bragg-Brentano geometry and PLXCEL 1D detector equipped with a nickel filter.

\section{UPS measurements.}

UPS spectra of the perovksite films were measured on Au coated substrate. Photoelectron spectroscopy was performed in a PHI5500 Multi-Technique system using non-monochromatized He-I $\alpha$ radiation (UPS) $(h v=21.22 \mathrm{ev})$. All work function and valence-band measurement were performed at a takeoff angle of $88^{\circ}$, with chamber pressure near $10^{-9}$ Torr.

Photoluminescence (PL) measurements. 
Photoluminescence (PL) measurements were performed using a Horiba Fluorolog system. Steady-state PL was collected by illuminating the samples with a monochromatized Xe lamp. Transient PL was acquired with a Time Correlated Single Photon Counting detector and a pulsed UV laser diode $(\lambda=375 \mathrm{~nm})$. The instrument response function provides an overall time resolution of $\Delta t \sim 0.13 \mathrm{~ns}$. Time-resolved emission spectra were mapped by collecting individual transient PL traces at different emission wavelengths. Absolute PL quantum yield (PLQY) measurements were done by coupling a Quanta-Phi integrating sphere to the Fluorolog system with optical fiber bundles. The measurements were done in accordance with published methods ${ }^{27}$. Both excitation and emission spectra were collected for the three cases of the sample directly illuminated by the excitation beam path in the integrating sphere, the sample offset within the integrating sphere from the beam path, and the empty sphere itself. The PLQY measurements were done by setting the Fluorolog to an excitation wavelength of $400 \mathrm{~nm}$ and to have a $5 \mathrm{~nm}$ bandpass for both the excitation and emission slits. With these settings, the ensuing spectra had high signal to noise ratios and delivered an excitation intensity in a range of 1-30 $\mathrm{mW} / \mathrm{cm}^{2}$ to the sample. Excitation intensity spectra were collected with a calibrated neutral density filter with known transmission placed after the integrating sphere. A Newport white light source was used to calibrate the detector and integrating sphere for spectral variance. PL spectra were collected as a function of excitation intensity by varying the slit width on the Fluorolog monochromator. The excitation intensity was calculated by measuring the power with an Ophir LaserStar Dual Channel Power and energy meter and calculating the beam area through the known dispersion relations for the monochromator.

\section{Transient absorption (TA) measurements.}


A regeneratively amplified $\mathrm{Yb}: \mathrm{KGW}$ laser at a $5 \mathrm{kHz}$ repetition rate (Light Conversion, Pharos) was used to generate femtosecond laser pulses, and a pulse picker was used to lower the frequency to $1 \mathrm{kHz}$. A portion of the $1030 \mathrm{~nm}$ fundamental was sent into an optical bench (Ultrafast, Helios), where it passed through a retroreflector, and was then focused into a calcium fluoride crystal, translated at $1 \mathrm{~mm} / \mathrm{s}$, to create the white light continuum probe. An optical parametric amplifier (Light Conversion, Orpheus) was used to generate the $350 \mathrm{~nm}$ pump pulse by upconversion of the fundamental wavelength. This was then sent into the optical bench and was chopped at $500 \mathrm{~Hz}$. Both the pump and probe were sent to the sample, with the time delay adjusted by changing the pathlength of the probe (time resolution $\sim 350 \mathrm{fs}$ ). The probe pulse was then collected by a CCD after dispersion by a grating spectrograph (Ultrafast). Pump fluences were kept at $20 \mu \mathrm{J} / \mathrm{cm}^{2}$. Kinetic traces were fit to the convolution of the instrument response and a sum of exponential decays. Time zero was allowed to vary with wavelength to account for the chirp of the probe. All TA measurement results were plotted based on the ref ${ }^{28}$. The relative presence of each domain (Figures 2 and 3) was quantified by the amplitude of the transient absorption signal at $t=0$.

\section{Device characterization}

The luminance (L) - current density $(\mathrm{J})$ - voltage (V) characteristics were collected by using a HP4140B picoammeter, a calibrated luminance meter (Konic Minolta LS-110). The absolute EL power spectra of the devices were collected with an integrating sphere and an Ocean Optics USB4000 spectrometer by mounting the devices on the wall of the integrating sphere. The EQE was then calculated through the measured absolute power spectra and current density.

\section{Calculations}


The procedure describing the applied stochastic Monte Carlo simulations is detailed in suppl. section S1. The ratio of excitons and free carriers in perovskite systems has been calculated based on ref $^{29}$. The PLQY as a function of power and funneling factor was obtained from:

$$
\operatorname{PLQY}\left(f, P_{\text {pump }}\right)=\frac{k_{\text {rad }}}{k_{\text {rad }}+k_{\text {trap }}+k_{\text {Auger }}}
$$

where for simplicity the $k_{\text {Auger }}$ term has been included.

Flat and engineered energy landscape modeling: The energy evolution in plain landscape materials was evaluated according to the next parameters: $A=2 \cdot 10^{9} \mathrm{~s}^{-1}, B=10^{8} \mathrm{~cm}^{3} \mathrm{~s}^{-1}$ and $C=$ $1.6 \cdot 10^{6} \mathrm{~cm}^{6} \mathrm{~s}^{-1}$, excited using a $150 \mathrm{fs}$ pump excitation of $20 \mu \mathrm{J} / \mathrm{cm}^{2}$. For engineered energy landscape films, the individual recombination rates of the $n_{\mathrm{i}}$ domains have been kept equal to those typical of the single material ${ }^{31}$. A transfer rate of $k_{\text {transfer }}=10^{11} \mathrm{~s}^{-1}$ was employed and the contribution of each domain weighted by $w=(0.6,0.3,0.1)$ and $w=(0.2,0.5,0.3)$ for the graded sample. 


\section{Acknowledgements}

This publication is based in part on work supported by Award KUS-11-009-21, made by King Abdullah University of Science and Technology (KAUST), by the Ontario Research Fund Research Excellence Program, and by the Natural Sciences and Engineering Research Council (NSERC) of Canada. L. N. Quan and D. H. Kim acknowledge the financial support by National Research Foundation of Korea Grant funded by the Korean Government (2014R1A2A1A09005656). F. P. García de Arquer acknowledges financial support from the Connaught fund. The authors thank H. Tan and X. Gong for their help during the course of study.

\section{Author Contributions}

L.Q., Y.Z., F.P.G.A and E.H.S. conceived the idea and proposed the experimental design. L.Q., F.P.G.A., R.S., G.W., O.V., R.C., Y.L., J.F., P.J., M.Y., and D.H.K. performed and analyzed XRD, UV absorption, PL lifetime, transient absorption and UPS measurements. L.Q. Y.Z. performed the device fabrication. F.P.G.A performed the simulation. L.Q., Y. Z. and Z. Lu tested the devices. L.Q., F.P.G.A., O.V. and E.H.S. co-wrote the manuscript. All authors read and commented on the manuscript. 

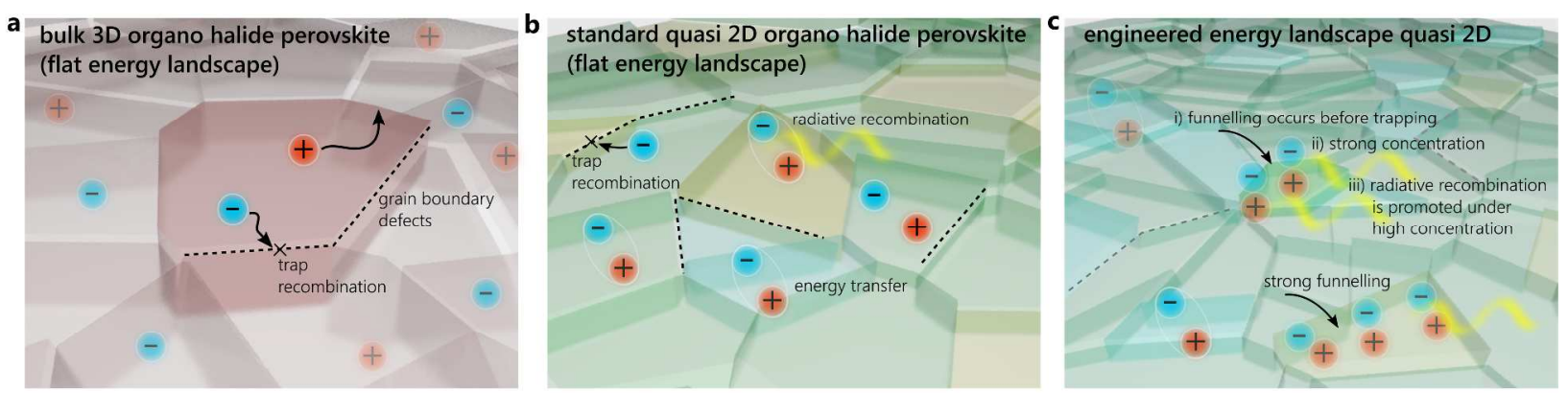

d

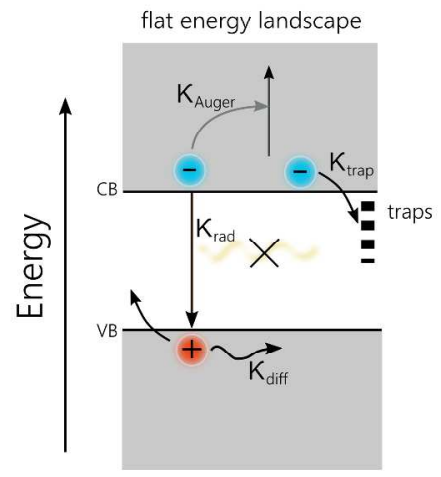

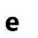

engineered energy landscape

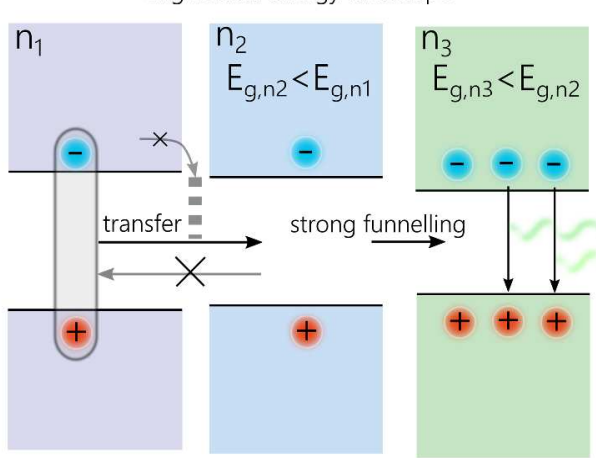

h
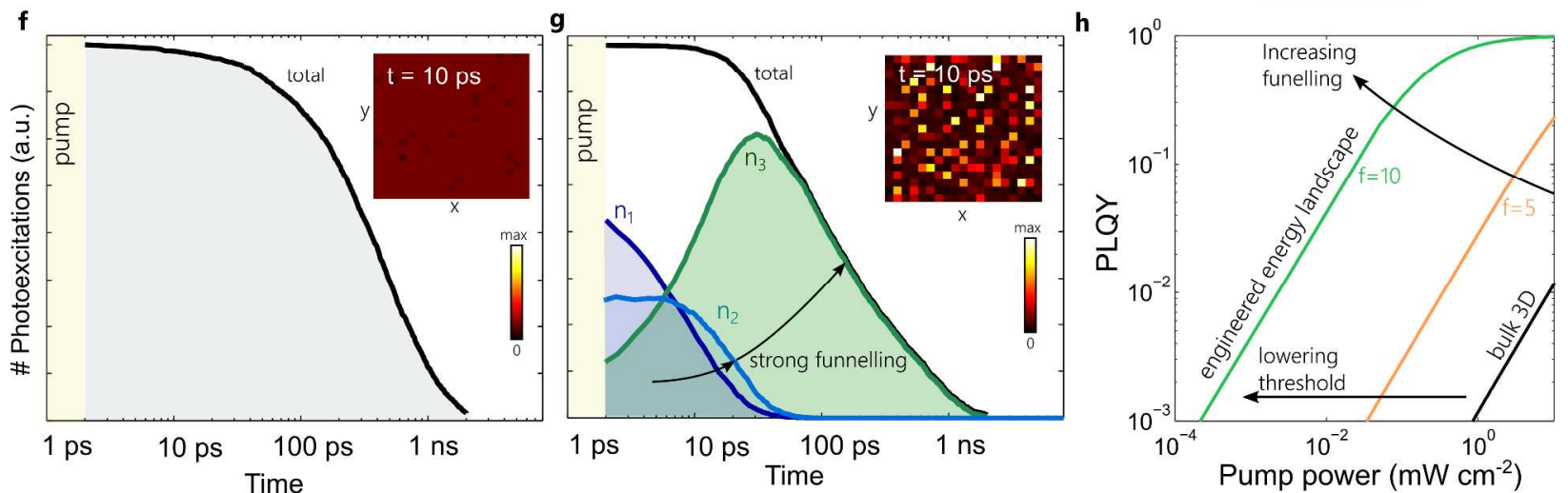

Figure 1. Tailoring the energy landscape in quasi-2D halide perovskites for efficient light emission. (a) In bulk-3D perovskites, the high diffusivity of charges militate against radiative recombination. (b) In quasi-2D perovskites, free-charges and excitons coexist. A competition between radiative and non-radiative recombination, exists determined by density of defects and on the density of photoexcited charges and excitons. (c) In energy-landscape-engineerd quasi-2D perovskites, energy funnelling occurs faster than non-radiative recombination. As a consequence, photoexcited states are strongly concentrated in a subpopulation of domains, thereby increasing the probability of bimolecular radiative recombination. (d) In a flat energy landscape material, charges can either be trapped (with a rate $\left.k_{\text {trap }}\right)$, or lost via Auger $\left(k_{\text {Auger }}\right)$ or radiative $\left(k_{\text {rad }}\right)$ recombination. The presence of trap states within, and at the boundaries, of the different grains, 
results in significant non-radiative recombination. (e) This limiting process can be overcome provided energy is funnelled to lower-bandgap domains before non-radiative recombination occurs. (f) Monte-Carlo simulation of the time evolution of photoexcited states after pumping in a flat energy landscape system. Inset: distribution of excited states within the organo halide perovskite matrix after the initial $10 \mathrm{ps}$. After initial excitation only trapping events are recorded. (g) In energy-landscape-engineered systems, energy transfer outcompetes non-radiative processes. The photoexcited states are fully transferred from $n_{1}$ to $n_{2}$ and $n_{3}$ domains within the first 100 ps. Inset: distribution of excited states after the initial 10 ps. An increasing contribution of radiative events is recorded upon exciton funneling (Fig S1). (h) The strong energy concentration in energy-landscape-engineered quasi-2D systems enabler high photoluminescence quantum yield at much lower pump intensities. 
a

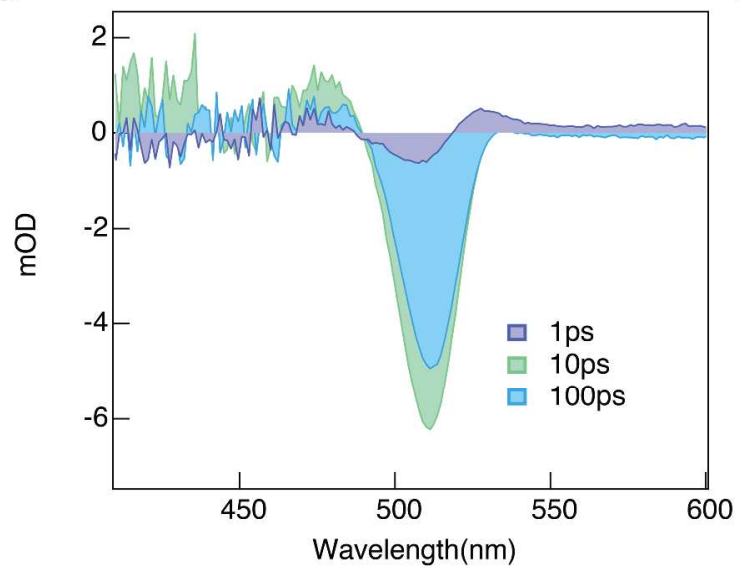

b

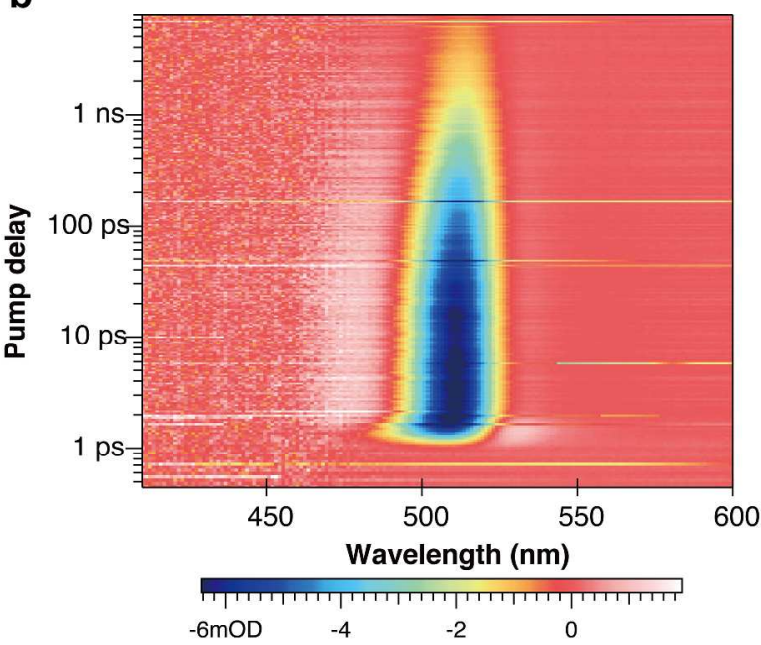

C

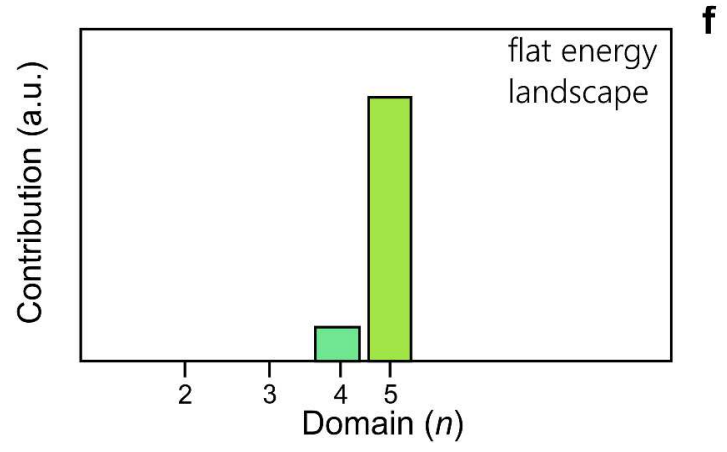

d

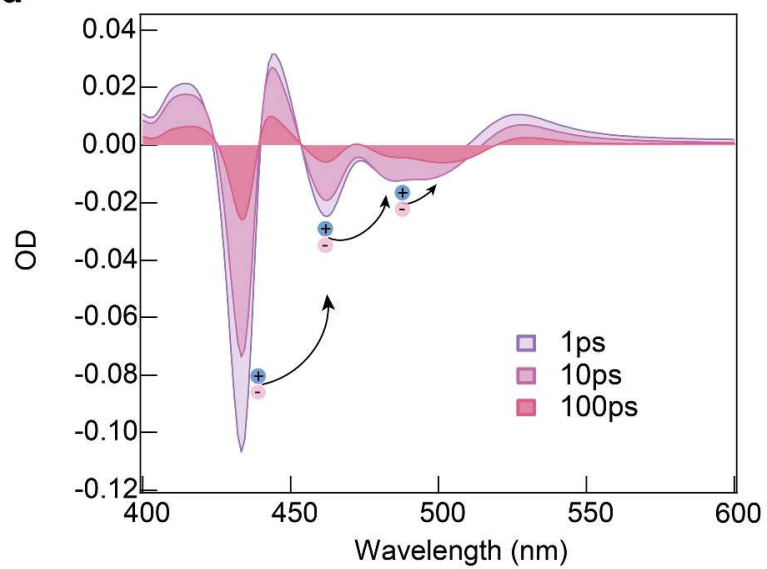

e
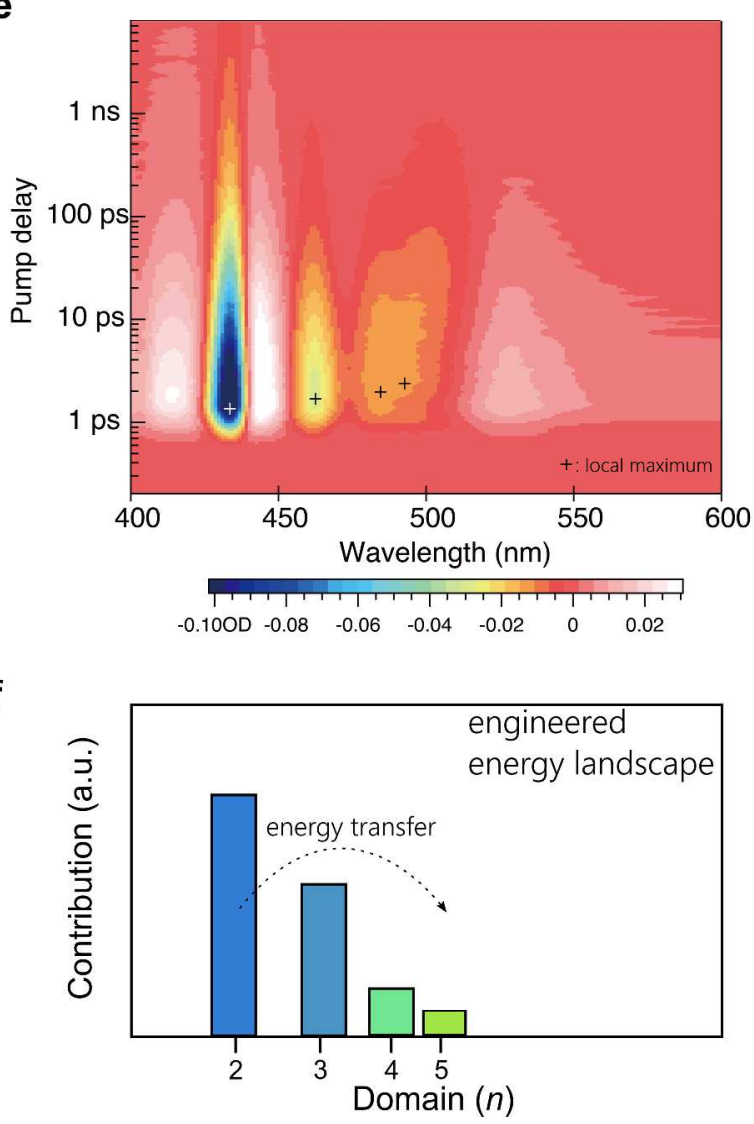

Figure 2 | Controlling the energy landscape for quasi-2D perovskites through the crystallization process. Transient absorption spectra of flat (a-c) and (d-f) energy-landscapeengineered $\langle n>=3$ quasi-2D perovskite films. The relative presence of different $n$ domains was extracted from the amplitude of the ground state bleach peaks (see methods). In the case of a flat energy landscape, $n=5$ dominates. Energy-landscape-engineered films, instead, consist of a set of different domains where energy funnels into the final $n=5$ domain. 
a
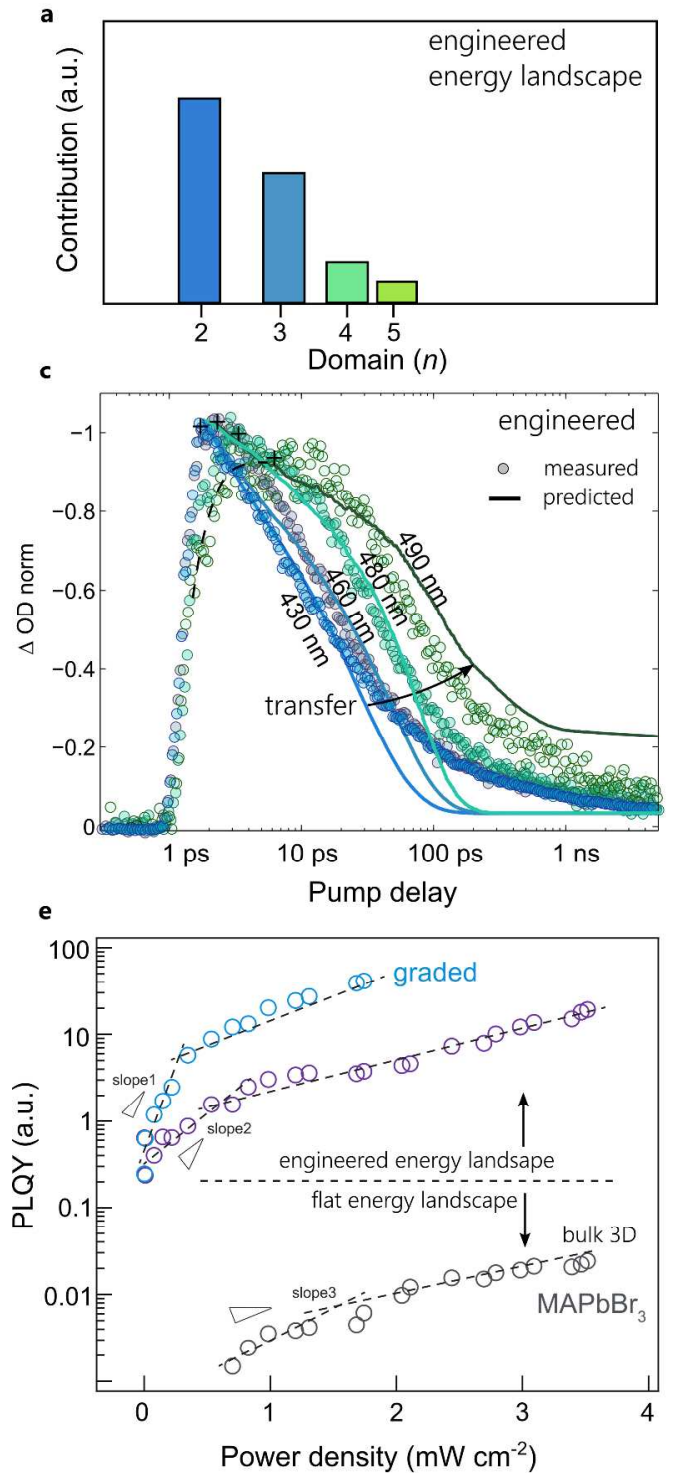

b

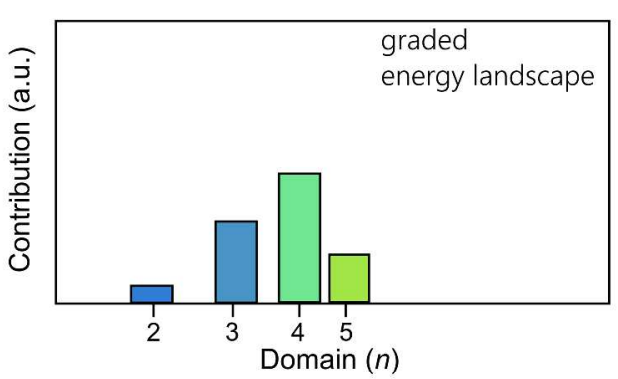

d

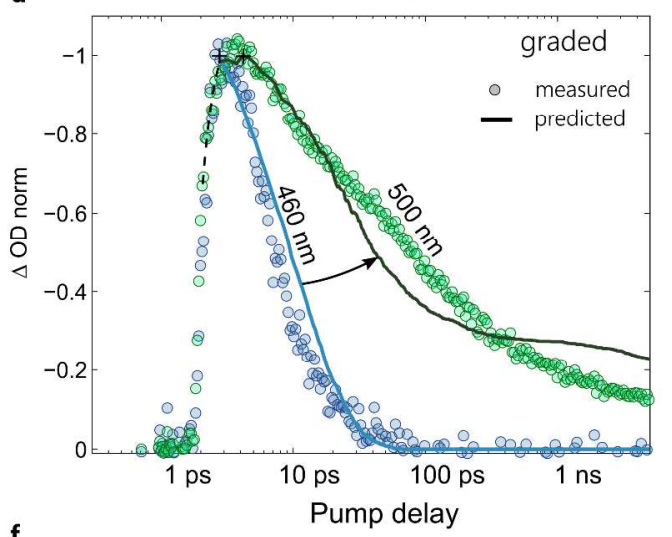

f

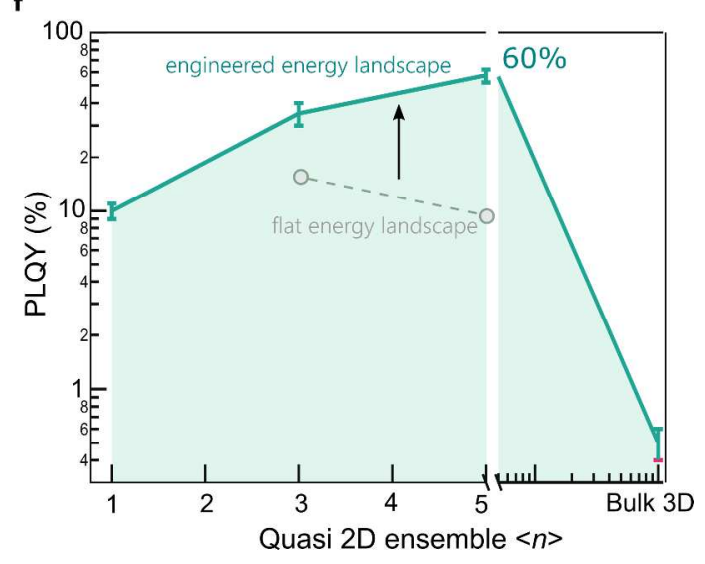

Figure 3 | Optimizing energy transfer in energy-landscape-engineered quasi-2D perovskite systems. (a-d) Contribution of different domains in energy-landscape-engineered and gradedenergy-landscape quasi-2D perovskite films as estimated from transient absorption spectroscopy (c-d) Experimental and predicted time dependent transient absorption traces for engineered $<n>=3$ (c) and graded $<n>=5$ (d) films for different wavelengths. (e) PLQY as a function of pump power density for different material systems, showcasing the benefits of energy-landscapeengineered quasi-2D perovskites. (f) PLQY for different quasi-2D perovskite configurations. 
a
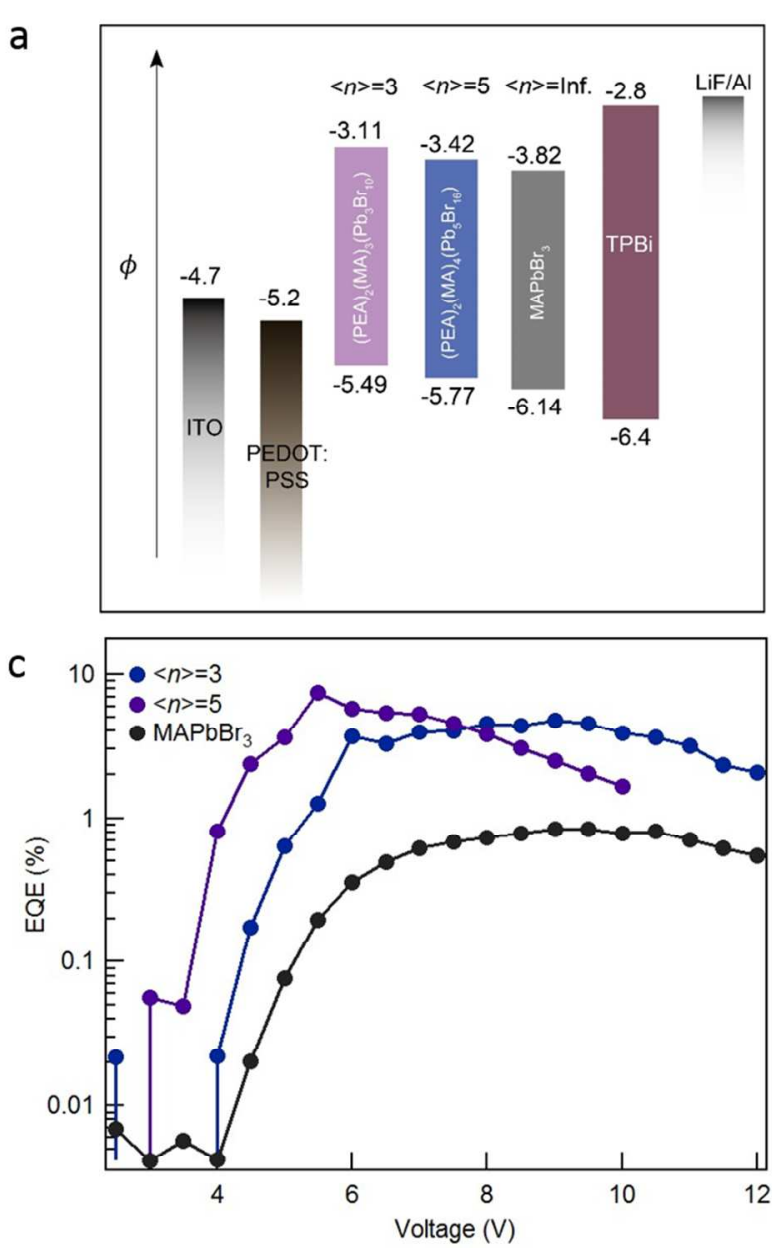

b
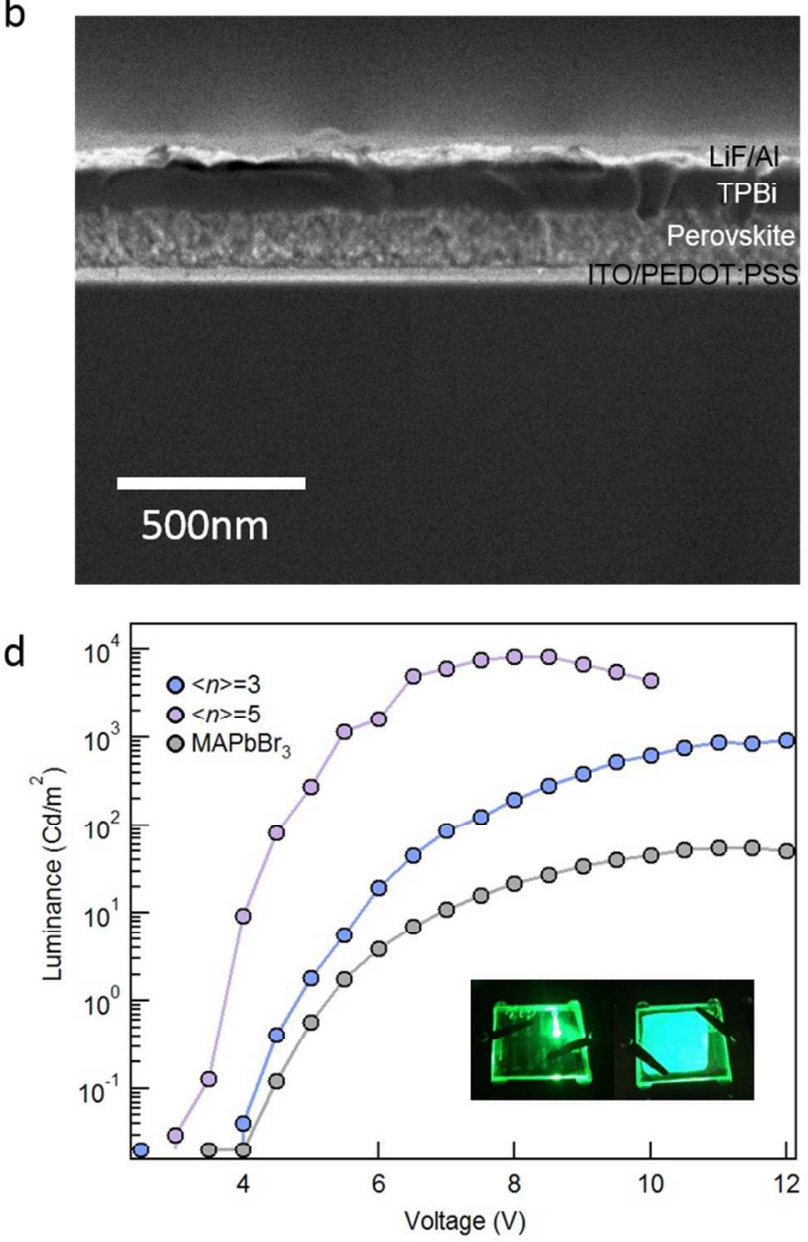

Figure 4 | Energy-landscape-engineered quasi-2D perovskites for highly efficient LEDs (a) Band structure of perovskites with different perovskites and energy band alignment of LED device. (b) Cross-sectional SEM image of LED device. (c) EQE versus applied voltage characteristics of the device with different perovskites. (d) Summary of the luminance in different perovskites based device. 
Table 1. Performance metrics for best perovskite-based light-emitting devices

\begin{tabular}{|c|c|c|c|c|c|}
\hline Ref. & Year/month & Emission material & $\begin{array}{c}\text { Emission } \\
\text { wavelength } \\
(\mathrm{nm})\end{array}$ & $\begin{array}{c}\text { Maximum } \\
\text { luminance } \\
\left(\mathrm{cd} / \mathrm{m}^{2}\right)\end{array}$ & $\begin{array}{c}\text { Maximum } \\
\text { EQE }(\%)\end{array}$ \\
\hline Tan et al. (7) & $2014 / 08$ & $\mathrm{MAPbBr}_{3}$ & 517 & 364 & 0.1 \\
\hline $\begin{array}{l}\text { Kim et al. } \\
\text { (9) }\end{array}$ & $2014 / 11$ & $\mathrm{MAPbBr}_{3}$ & 543 & 417 & 0.125 \\
\hline Li et al. (11) & $2015 / 01$ & $\mathrm{MAPbBr}_{3}$ & 534 & 200 & 1.2 \\
\hline $\begin{array}{c}\text { Cho et al. } \\
(10)\end{array}$ & $2015 / 12$ & $\mathrm{MAPbBr}_{3}$ & 540 & 10000 & 8.53 \\
\hline $\begin{array}{c}\text { Byun et al. } \\
\text { (24) }\end{array}$ & $2016 / 06$ & $\left(\mathrm{MAPbBr}_{3}\right)\left(\mathrm{PEA}_{2} \mathrm{PbBr}_{4}\right)_{6}$ & 510 & 2935 & - \\
\hline $\begin{array}{c}\text { Wang et al. } \\
\text { (23) }\end{array}$ & $2016 / 09$ & $(\mathrm{NMABr})_{2}(\mathrm{FABr})\left(\mathrm{PbBr}_{2}\right)_{2}$ & 518 & - & 0.1 \\
\hline This work & 2016 & $\mathrm{PEA}_{2}(\mathrm{MA})_{4} \mathrm{~Pb}_{5} \mathrm{Br}_{16}$ & 520 & 8400 & 7.4 \\
\hline
\end{tabular}

Table 2. TA (Experimental) lifetime

\begin{tabular}{|c|c|c|c|c|c|c|}
\hline \multicolumn{3}{|c|}{$<n>=3$} & \multicolumn{3}{c|}{$<n>5$} \\
\hline & $430 \mathrm{~nm}$ & $460 \mathrm{~nm}$ & $480 \mathrm{~nm}$ & $490 \mathrm{~nm}$ & $460 \mathrm{~nm}$ & $500 \mathrm{~nm}$ \\
\hline $\begin{array}{c}1^{\text {st }} \\
\text { exponential }\end{array}$ & $5.7 \mathrm{ps}$ & $16 \mathrm{ps}$ & $30.7 \mathrm{ps}$ & $70.5 \mathrm{ps}$ & $3.5 \mathrm{ps}$ & $6.07 \mathrm{ps}$ \\
\hline $\begin{array}{c}2^{\text {nd }} \\
\text { exponential }\end{array}$ & $42 \mathrm{ps}$ & $198 \mathrm{ps}$ & $169 \mathrm{ps}$ & $1 \mathrm{~ns}$ & $26 \mathrm{ps}$ & $3 \mathrm{~ns}$ \\
\hline
\end{tabular}

Table 3. PLQY, pump power $\left(\boldsymbol{P}_{\text {pump }}\right)$ and PLQY-to- $\boldsymbol{P}_{\text {pump }}$

\begin{tabular}{|c|c|c|c|c|c|c|}
\hline Ref. & Year & Regime & $\begin{array}{c}\text { PLQY } \\
(\%)\end{array}$ & $\begin{array}{c}P_{\text {pump }} \\
(\mathrm{mW} \mathrm{cm})^{-2}\end{array}$ & $\begin{array}{c}\text { PLQY/ } \\
P_{\text {pump }}\end{array}$ & Perovskite \\
\hline 15 & 2014 & NIR & 50 & 100 & 0.005 & $\mathrm{MAPbI}_{\mathrm{x}} \mathrm{Cl}_{3-\mathrm{x}}$ \\
\hline 7 & 2014 & NIR & 26 & 333 & 0.00078 & $\mathrm{MAPbI}_{\mathrm{x}} \mathrm{Cl}_{3-\mathrm{x}}$ \\
\hline 7 & 2014 & Visible & 7 & - & - & $\mathrm{MAPbBr}_{3}$ \\
\hline 10 & 2015 & Visible & 36 & 1000 & 0.00036 & $\mathrm{MAPbBr}_{3}$ \\
\hline $\begin{array}{c}\text { Flat energy } \\
\text { landscape } \\
\text { (This work) }\end{array}$ & 2016 & Visible & 12 & 1.8 & 0.06 & $\mathrm{PEA}_{2}(\mathrm{MA})_{4} \mathrm{~Pb}_{5} \mathrm{Br}_{16}$ \\
\hline $\begin{array}{c}\text { Engineered } \\
\text { landscape } \\
\text { This work }\end{array}$ & $\mathbf{2 0 1 6}$ & Visible & $\mathbf{6 0}$ & $\mathbf{1 . 8}$ & $\mathbf{0 . 3 3}$ & $\mathbf{P E A}_{\mathbf{2}}\left(\mathbf{M A}_{\mathbf{4}} \mathbf{P b}_{\mathbf{5}} \mathbf{B r}_{\mathbf{1 6}}\right.$ \\
\hline
\end{tabular}




\section{References}

1. Lee MM, Teuscher J, Miyasaka T, Murakami TN, Snaith HJ. Efficient hybrid solar cells based on meso-superstructured organometal halide perovskites. Science 338, 643-647 (2012).

2. Burschka J, et al. Sequential deposition as a route to high-performance perovskitesensitized solar cells. Nature 499, 316-319 (2013).

3. Jeon NJ, et al. Compositional engineering of perovskite materials for high-performance solar cells. Nature 517, 476-480 (2015).

4. Xing G, et al. Low-temperature solution-processed wavelength-tunable perovskites for lasing. Nat Mater 13, 476-480 (2014).

5. Sutherland BR, Hoogland S, Adachi MM, Wong CT, Sargent EH. Conformal organohalide perovskites enable lasing on spherical resonators. ACS Nano 8, 1094710952 (2014).

6. Zhu $\mathrm{H}$, et al. Lead halide perovskite nanowire lasers with low lasing thresholds and high quality factors. Nature Materials 14, 636-642 (2015).

7. Tan Z-K, et al. Bright light-emitting diodes based on organometal halide perovskite. Nature Nanotechnology 9, 687-692 (2014).

8. Hoye RL, et al. Enhanced Performance in Fluorene - Free Organometal Halide Perovskite Light - Emitting Diodes using Tunable, Low Electron Affinity Oxide Electron Injectors. Advanced Materials 27, 1414-1419 (2015).

9. Kim YH, et al. Multicolored Organic/Inorganic Hybrid Perovskite Light - Emitting Diodes. Advanced Materials 27, 1248-1254 (2015).

10. Cho $\mathrm{H}$, et al. Overcoming the electroluminescence efficiency limitations of perovskite light-emitting diodes. Science 350, 1222-1225 (2015).

11. Li G, et al. Efficient light-emitting diodes based on nanocrystalline perovskite in a dielectric polymer matrix. Nano Letters 15, 2640-2644 (2015).

12. Hong X, Ishihara T, Nurmikko AV. Dielectric confinement effect on excitons in PbI 4based layered semiconductors. Physical Review B 45, 6961 (1992).

13. Tanaka K, Takahashi T, Ban T, Kondo T, Uchida K, Miura N. Comparative study on the excitons in lead-halide-based perovskite-type crystals $\mathrm{CH}_{3} \mathrm{NH}_{3} \mathrm{PbBr}_{3} \mathrm{CH}_{3} \mathrm{NH}_{3} \mathrm{PbI}_{3}$. Solid State Communications 127, 619-623 (2003). 
14. Yang Y, Yang M, Li Z, Crisp R, Zhu K, Beard MC. Comparison of recombination dynamics in $\mathrm{CH}_{3} \mathrm{NH}_{3} \mathrm{PbBr}_{3}$ and $\mathrm{CH}_{3} \mathrm{NH}_{3} \mathrm{PbI}_{3}$ perovskite films: Influence of exciton binding energy. The Journal of Physical Chemistry Letters 6, 4688-4692 (2015).

15. Deschler F, et al. High photoluminescence efficiency and optically pumped lasing in solution-processed mixed halide perovskite semiconductors. The Journal of Physical Chemistry Letters 5, 1421-1426 (2014).

16. Stranks SD, Burlakov VM, Leijtens T, Ball JM, Goriely A, Snaith HJ. Recombination kinetics in organic-inorganic perovskites: excitons, free charge, and subgap states. Physical Review Applied 2, 034007 (2014).

17. Saparov B, Mitzi DB. Organic-Inorganic Perovskites: Structural Versatility for Functional Materials Design. Chem Rev 116, 4558-4596 (2016).

18. Quan LN, et al. Ligand-stabilized reduced-dimensionality perovskites. Journal of the American Chemical Society 138, 2649-2655 (2016).

19. $\mathrm{Yu} \mathrm{JC}$, et al. Improving the Stability and Performance of Perovskite Light - Emitting Diodes by Thermal Annealing Treatment. Advanced Materials, (2016).

20. Mitzi DB, Feild C, Harrison W, Guloy A. Conducting tin halides with a layered organicbased perovskite structure. Nature 369, 467-469 (1994).

21. Sichert JA, et al. Quantum size effect in organometal halide perovskite nanoplatelets. Nano Letters 15, 6521-6527 (2015).

22. Milot RL, et al. Charge Carrier Dynamics in 2D Hybrid Metal Halide Perovskites. Nano Letters, (2016).

23. Wang $\mathrm{N}$, et al. Perovskite light-emitting diodes based on solution-processed selforganized multiple quantum wells. Nature Photonics, (2016).

24. Byun J, et al. Efficient Visible Quasi - 2D Perovskite Light - Emitting Diodes. Advanced Materials 28, 7515-7520 (2016).

25. Yuan M, et al. Perovskite energy funnels for efficient light-emitting diodes. Nature Nanotechnology, (2016).

26. Wu X, et al. Trap states in lead iodide perovskites. Journal of the American Chemical Society 137, 2089-2096 (2015).

27. Sum TC, et al. Spectral Features and Charge Dynamics of Lead Halide Perovskites: Origins and Interpretations. Accounts of Chemical Research 49, 294-302 (2016). 
28. Manser JS, Kamat PV. Band filling with free charge carriers in organometal halide perovskites. Nature Photonics 8, 737-743 (2014).

29. Jeon NJ, Noh JH, Kim YC, Yang WS, Ryu S, Seok SI. Solvent engineering for highperformance inorganic-organic hybrid perovskite solar cells. Nature Materials 13, 897903 (2014).

30. Berera R, van Grondelle R, Kennis JT. Ultrafast transient absorption spectroscopy: principles and application to photosynthetic systems. Photosynthesis Research 101, 105118 (2009).

31. Rowland CE, et al. Picosecond energy transfer and multiexciton transfer outpaces Auger recombination in binary CdSe nanoplatelet solids. Nature Materials 14, 484-489 (2015). 